\title{
CONDUCTA INFORMATIVA DE LOS INVESTIGADORES ARGENTINOS EN HUMANIDADES Y CIENCIAS SOCIALES
}

\section{Susana Romanos de Tiratel*}

Resumen: Se expone una investigación que intenta responder a dos cuestiones: ies correcto transferir las conclusiones de los estudios de usuarios en $\mathrm{Hu}$ manidades y Ciencias Sociales llevados a cabo en los países anglosajones al medio universitario argentino que presenta realidades diferentes, tiene ambientes organizacionales distintos y sistemas y servicios bibliotecarios menos desarrollados? Y, si a pesar de las disimilitudes de recursos, existe un sustrato común que tipifica al investigador en Humanidades y en Ciencias Sociales respecto de sus actividades de acceso a la información, sus preferencias y sus necesidades informativas, independientemente del lugar donde desarrolle sus tareas y de las facilidades materiales de las que disponga. A partir de los problemas planteados se fijo como objetivo establecer perfiles, modalidades de acceso y preferencias en la utilización de la información de los investigadores en Humanidades y Ciencias Sociales de la Facultad de Filosofía y Letras (UBA). Una vez hecho esto, comprobar similitudes y diferencias con los resultados de investigaciones similares efectuadas, principalmente, en los países anglosajones. Se utilizó una metodología que combinó encuestas, entrevistas y análisis de citas. Los resultados llevan a concluir que, en primer lugar, no existen diferencias sustanciales en la conducta informativa de los investigadores de ambas áreas entre sí, ni tampoco marcadas disimilitudes con la que se ha descrito en otros países; por lo tanto, las dos hipótesis iniciales se han visto confirmadas por los resultados de este estudio.

Palabras clave: estudios de usuarios; Humanidades; Ciencias Sociales; conducta informativa; acceso a la información; Argentina.

Abstract: The reported research intends to answer two questions: 1.Can we transfer conclusions made in studies of Humanities and Social Sciences users in Anglo Saxon countries to an Argentine university milieu, with different realities, different organizations, and less developed library systems and services? 2. Even though resources are different, is there a common background characterizing the Humanities and Social Sciences researchers as to activities related to access to information, preferences, and information needs regardless the place where they work and material facilities available? The goal was to develop profiles, access styles and preferences in using information by Humanities and Social Sciences researchers at the $\mathrm{Fa}$ cultad de Filosofía y Letras (Universidad de Buenos Aires). Then prove similarities and differences with similar results in investigations undertaken mainly in Anglo Saxon countries. Methodology used combined questionnaires, interviews and citation analysis. The conclusions are: first, there are no substantial differences between information behaviour of researchers in

* Instituto de Investigaciones Bibliotecológicas. Facultad de Filosofía y Letras, Universidad de Buenos Aires. Argentina. Correo-e: inibi@filo.uba.ar.

Recibido: 3-11-99. 
both areas, nor are there differences in behaviour as described in other countries. Therefore, in this study both initial hypotheses have been confirmed by results.

Keywords: user studies; Humanities; Social Sciences; information behaviour; information access; Argentina.

\section{Introducción}

En la literatura anglosajona de Bibliotecología/Ciencia de la Información existe un número considerable de estudios de usuarios que describen las necesidades, usos y conducta de búsqueda de la información de los investigadores en Ciencias Sociales y en Humanidades. Se encuentran, además, revisiones que sintetizan e integran las conclusiones derivadas de estos estudios, constituyéndose en listas de características que describen y tipifican a los investigadores de esas áreas.

Durante años, los profesores de Bibliotecología/Ciencia de la Información de Iberoamérica han proporcionado a sus alumnos retratos más o menos precisos de los investigadores en Humanidades y Ciencias Sociales, basándose sobre los ya mencionados hallazgos de investigación hechos en Estados Unidos y Gran Bretaña. La primera pregunta que se plantea es una derivación lógica de la situación descrita, ies correcto transferir las conclusiones de esos estudios a realidades diferentes, con ambientes distintos y con sistemas y servicios bibliotecarios menos desarrollados? La segunda cuestión a considerar es si, a pesar de las diferencias, existen modos de trabajo y conductas informativas que trascienden la diversidad, dado que son propias de las personas en la situación cognoscitiva, influidas por la educación y por lo que podría denominarse la «cultura» de las diferentes áreas de estudio y de los ambientes de la organización en la que se desempeñan, aunque diversificadas por los antecedentes propios de cada individuo. Esto es, si a pesar de las disimilitudes de recursos, existe un substrato común que tipificaría al investigador en Humanidades y en Ciencias Sociales en sus actividades de acceso a la información, en sus preferencias y en sus necesidades informativas, independientemente del lugar donde desarrolle sus tareas y de las facilidades materiales de las que disponga.

A partir de los problemas planteados, esta investigación se propuso establecer perfiles, modalidades de acceso y preferencias en la utilización de la información de los investigadores en Humanidades y Ciencias Sociales de la Facultad de Filosofía y Letras (UBA). Una vez hecho esto, comprobar similitudes y diferencias con los resultados de investigaciones similares efectuadas, principalmente, en los países anglosajones. En segundo término, se ponen a disposición de la comunidad profesional los resultados que trazan un perfil de los investigadores, con el propósito de facilitar la evaluación y desarrollo de las colecciones de las bibliotecas universitarias de investigación y especializadas, y el diseño de sistemas y servicios adaptados a la conducta informativa de los estudiosos en ambas áreas.

En la Argentina, no existe una línea de investigación continua dedicada a los estudios de usuarios. A fines de la década de los 60, en el Centro de Investigaciones Bibliotecológicas de la Facultad de Filosofía y Letras (UBA), Cirigliano (1) hizo un estudio de la conducta informativa de estudiantes universitarios avanzados y recién 
egresados, sin distinguir entre disciplinas o áreas de estudio. Se tiene conocimiento de un estudio, muy poco difundido, de los científicos políticos argentinos realizado en 1977 , cuyos resultados se incorporaron como parte de una tesis de doctorado (2). Hasta 1995, en que se inicia un nuevo programa de estudios de usuarios del que se informa en este artículo, no se registran otras investigaciones publicadas sobre el tema en la Argentina.

Considerando esta situación, los interrogantes planteados y las características más frecuentes mencionadas por los estudios existentes, se diseñó un proyecto de investigación para estudiar a un grupo de investigadores en Humanidades y Ciencias Sociales de la Facultad de Filosofía y Letras de la Universidad de Buenos Aires, con el propósito de averiguar cómo encuentran la información necesaria no sólo para mantenerse al día en los avances de sus respectivas disciplinas sino también para apoyar sus tareas de investigación; cuál es el formato preferido de la literatura que usan: libros o revistas; cuál es la tasa de obsolescencia del material que manejan; cuál es el grado de dispersión temática de la información que necesitan; y cuál es la lengua de preferencia de la bibliografía consultada. De este modo, una vez obtenidos estos datos, se podría saber si hay diferencias entre ambos campos disciplinarios, cuáles son las disparidades $\mathrm{y}$, finalmente, analizar las coincidencias o divergencias con los resultados de estudios realizados en otros países.

Esta investigación sólo pretendió describir un estado de situación en un momento dado, para poder enfocar, posteriormente, con mayor profundidad, aspectos más puntuales surgidos en esta primera etapa. Fue desarrollada, en el término de tres años (1995-1997), por un grupo constituido por profesores y graduados de la Carrera de Bibliotecología y Documentación (*). Se estudió a los profesores e investigadores de la Facultad que imparte nueve carreras dedicadas tanto a las Humanidades como a las Ciencias Sociales: Filosofía, Letras, Lenguas y Literaturas Clásicas, Artes, Historia, Ciencias Antropológicas, Ciencias de la Educación, Geografía y Bibliotecología.

La estructura académica de la Facultad está constituida por nueve Departamentos, que atienden al desarrollo de los programas de grado (profesorados y licenciaturas), por una Secretaría de Postgrado que apoya los estudios de postgrado (doctorado, maestrías, carreras de especialización y actualización) y por 20 institutos donde se elaboran y llevan a cabo los proyectos y programas de investigación.

Las unidades de información se componen de una Biblioteca Central, con colecciones que sirven, fundamentalmente, a los estudios de grado y de postgrado. Funcionan, además, dieciocho bibliotecas de investigación con sede en los institutos, que gestionan colecciones especializadas en la temática específica que se desarrolla en cada uno de éstos.

Dado que la unidad de análisis de esta investigación son los investigadores, para llevarla a cabo se eligieron - por su importancia tanto cuantitativa como cualitativalos institutos más representativos de las disciplinas que se desarrollan en la Facultad. En estos se concentran los docentes/investigadores y los estudiantes y graduados con becas para iniciarse o perfeccionarse en la investigación, así como los recursos informativos que sustentan sus actividades. En este estudio no se incluyó al grupo de becarios estudiantes, por considerarlo menos representativo, dado que no todos continúan la carrera de docentes/investigadores. 


\section{Estado de la cuestión}

Los estudios de usuarios que se han llevado a cabo en otros países intentan determinar las características de la información y de sus usuarios, la relación existente entre los servicios de información y la estructura del conocimiento, cuáles son las necesidades y la utilización de la información en el área y, finalmente, cuál es la estructura de la literatura especializada: su tamaño, crecimiento y composición. Todas las investigaciones se han desarrollado, fundamentalmente, en Estados Unidos y Gran Bretaña.

Se revisará la literatura dedicada al estudio de los especialistas en Humanidades y de los científicos sociales en general, sin discriminar las disciplinas que conforman ambas áreas del conocimiento. De igual modo, el examen de la literatura producida no será exhaustivo sino que seleccionará, dentro de ésta, aquellas unidades de análisis que se corresponden con las que se han definido para esta investigación: acceso a la información, formato, obsolescencia de los materiales utilizados, dispersión temática y lengua; además, se preferirán artículos o libros que sinteticen e integren los hallazgos de los estudios realizados.

\subsection{Humanidades}

Watson-Boone (3) resume muy bien las modalidades de búsqueda de información cuando expresa que los humanistas tratan de elaborar una nueva interpretación de un tema, y que la especialización humanística tiene una naturaleza acumulativa y no recapitulätiva. De este modo, estos investigadores tienen una necesidad limitada de desarrollar o usar herramientas bibliográficas generales y otros servicios diferentes de información secundaria. Además de utilizar a los colegas, tienden a buscar dentro del material fuente impreso, inicial, referencias explícitas e implícitas a otros trabajos. Las reseñas de libros y las colecciones personales ocupan el lugar de los resúmenes e índices, de las guías y bases de datos. Se usan los catálogos de la biblioteca para encontrar materiales conocidos; pueden consultar a los bibliotecarios que trabajan en una colección que no les es familiar cuando necesitan documentos de los que sospechan su existencia en ese lugar. Además, el uso de algunos materiales primarios puede requerir que el investigador vaya directamente a éstos, dondequiera se localicen. Estos comentarios sugieren que la falta de adecuación de las herramientas de acceso y de los servicios secundarios puede no ser una cuestión de mucho interés para los investigadores del área. Y que lo que puede percibirse como una actitud de indiferencia refleje, en cambio, simplemente, una falta general de necesidad. El énfasis se pone sobre la interacción directa entre el estudioso y su material.

En cuanto al formato, Blazek y Aversa (4) afirman que la centralidad de la bi blioteca para el investigador en Humanidades aún se acompaña con la preferencia por la monografía en detrimento del artículo de revista. Stone (4), en su artículo que reseña las investigaciones publicadas entre 1970 y 1982, afirma que se daría un uso parejo de monografías y de artículos de publicaciones periódicas, dado que la evidencia respecto de qué formato se usa más es conflictiva. En este punto, Watson-Boone (3), cuando revisa dieciséis estudios puhlicados entre 1983 y 1992, concluye que la 
mayoría de los trabajos utilizados por los especialistas en Humanidades están en forma de libro. Si bien es cierta la presunción de que los libros desempeñan un papel más importante que las revistas, este aserto debería atemperarse, porque las materias y períodos cubiertos por el tema investigado determinan si el investigador usará un porcentaje mayor o menor de artículos y si el material monográfico serán las obras primarias (fuentes) del/de los individuo(s) estudiado(s) o la literatura crítica (secundaria).

Otro patrón de uso es la gran cantidad de tiempo de vigencia de los materiales. Los humanistas probablemente estén igualmente interesados en publicaciones de hace veinte, cuarenta o cincuenta años. Por cierto, si se consideran los «clásicos» en cada campo, la duración del interés puede extenderse hacia documentos de 2.000 o 3.000 años de antigüedad (4). Stone (4) concluye que el material original aparecido en cualquier fecha es más importante que el material actual. El valor de las publicaciones humanísticas se mantiene a través del tiempo, tal como lo afirman Blazek y Aversa (5) basándose en los estudios empíricos de citas. Sin embargo, Watson-Boone (3) es más precisa al respecto cuando sintetiza los resultados de las investigaciones examinadas, dado que las mismas clarifican que la mayoría de las citas y referencias primarias y secundarias se aglutinan alrededor de materiales que anteceden a la investigación en veinte a treinta años. Esto no implica que no se usen documentos más viejos, sino que lo más frecuente es que la mayoría de los materiales se encuentren dentro de este intervalo. Una vez más, el tema de la investigación dictará la extensión exacta (por ej., cuanto más antiguo es el tema, es más probable que el intervalo de la fecha de la cita sea mayor).

Respecto de la variedad de materias que usa el humanista en su investigación, es interesante consultar el artículo de Broadus (6) donde indica que 26 es el promedio de materias usadas por cada investigador estudiado en el National Humanities Center. Concluye que su estudio, basado en el uso real, sugiere que los materiales relevantes para la investigación en Humanidades están muy dispersos en las colecciones de la biblioteca, no sólo porque el esquema de clasificación fracasa en reunir ideas similares, sino porque los humanistas están interesados en ideas sobre temas muy diferentes. Otro ítem importante que aporta el estudio es que la amplia gama de materias utilizadas apoya el punto de vista de que, para los estudiosos de las Humanidades, la centralización de las colecciones es más práctica que la descentralización. Con anterioridad, ya había advertido Stone (4) que es imposible que una sola colección o biblioteca sea capaz de satisfacer totalmente las necesidades de los estudios humanísticos. De ahí que, al ser tan amplia la gama de materias y materiales requeridos por el investigador, el préstamo interbibliotecario sea crucial para las Humanidades.

En relación con la variable lengua, Broadus (7) indica que, en su estudio, el porcentaje de ítems en inglés fue notablemente más alto (82\%) que lo indicado por la mayoría de los estudios de citas en esta área del conocimiento (alrededor del $50 \%$ ). Esta preferencia, comprobada a través de las solicitudes de préstamo interbibliotecario, fue corroborada posteriormente por los mismos investigadores.

Cullars (8) cuando estudia las características de las citas en las monografías literarias francesas y alemanas comprueba porcentajes semejantes en referencia a las respectivas lenguas maternas (75\% para el alemán y $84 \%$ para el francés). El mismo autor (9) registra cantidades similares en artes plásticas cuando analiza las citas en las monografías de investigadores estadounidenses; el $76 \%$ están en inglés. 


\subsection{Ciencias Sociales}

En un artículo de revisión, Folster (10) pasa revista a diferentes métodos aplicados, durante los pasados 30 años, para estudiar el tipo de información que usan los investigadores en Ciencias Sociales y cómo la utilizan. Examina la literatura generada aplicando técnicas de investigación diversas: patrones de cita como medida del uso, cuestionarios, una combinación de técnicas de cuestionarios y entrevistas, u observación estructurada complementada con entrevistas.

Folster (10) afirma que las revistas son la fuente de información más importante en el proceso de investigación de los científicos sociales, dado que se convierten en un factor esencial en la adquisición de información porque se utilizan, en gran medida, para rastrear citas. Este aserto se ve corroborado por estudios previos citados por Baxter (11), donde se dan datos sobre las fuentes de información bibliográfica utilizadas en Ciencias Sociales. Las listas de citas agregadas a los libros o artículos de revistas y las secciones bibliográficas de las revistas se constituyen en las fuentes de citas más comunes $(69,7 \%$ y $65,1 \%$, respectivamente) y son las que se usan con más regularidad. Los autores concluyen que el acceso a la literatura relevante para futuras investigaciones se hace fundamentalmente a través de listas de citas publicadas previamente y en menor medida mediante contactos con colegas, artículos de reseña en libros y biblioteca personal.

Al revisar estudios anteriores, Baxter (11) concluye que los científicos sociales confían más en la comunicación personal y las listas de citas que los investigadores en los campos de las Ciencias Naturales y de la Técnica. Por ejemplo, sólo el $10 \%$ de las citas a publicaciones periódicas de Sociología fueron obtenidas de una fuente de índices o resúmenes, mientras que el $70 \%$ se obtuvo de una lista de citas o de recomendaciones verbales de un colega. Es importante tener en mente, sin embargo, la relativa dispersión de la cobertura provista por los índices disponibles comparados con los de las ciencias «duras» y aplicadas. Sin embargo, en 1995, Folster (10) coincide en gran medida cuando indica que se ha demostrado que consultar a colegas y expertos en el campo es un método valorizado de búsqueda de información. La comunicación informal juega un importante papel en el flujo de la información entre los científicos sociales. Las comunicaciones personales son una de las fuentes secundarias de información bibliográfica más importantes.

En cuanto al formato, los científicos sociales están muy orientados hacia lo impreso, ya sea que se trate de monografías o de publicaciones periódicas. Sobre la base de estudios previos, $\mathrm{Li}(12)$ destaca que los artículos de revistas se citan relativamente con menor frecuencia en Ciencias Sociales que en Ciencia y Técnica. Sin embargo, hay que tener en cuenta que la preferencia por uno $u$ otro formato varía entre las disciplinas del área $(12,13)$. De todos modos, se coincide en afirmar que hay una utilización pareja de libros y artículos de revistas. Los investigadores confían por igual en libros y en literatura periódica, lo que sugiere que los sistemas bibliográficos diseñados siguiendo los modelos de las Ciencias Físicas, donde predomina la literatura periódica, son de utilidad limitada en Ciencias Sociales $(10,13)$.

Otro patrón de uso es que los materiales actuales se usan intensamente. El uso de los materiales en las Ciencias Sociales es, sin embargo, prolongado y a menudo repetitivo. Según estudios citados por Li (12), la vida media de las citas totales en Ciencias Sociales es de nueve años, y las citas a publicaciones periódicas de seis años. 
Respecto de la variedad de materias que utiliza el científico social, el espectro de disciplinas sociales que cita es amplio. Li (12) indica que los hallazgos de las investigaciones revelan que, consideradas las Ciencias Sociales en conjunto, la auto cita (las Ciencias Sociales citando ítems de Ciencias Sociales), alcanza el 58\%, pero que la auto cita en una subdisciplina particular de las Ciencias Sociales es, generalmente, más baja. Comparada con la literatura usada en Ciencia y Tecnología, la self-deprivation (las Ciencias Sociales citando ítems de otros campos que contribuyen a las Ciencias Sociales) es apreciablemente más alta. De todos modos, los porcentajes varían entre disciplinas.

En relación con la variable lengua, $\mathrm{Li}$ (12), en el capítulo dedicado a enumerar las necesidades y usos bibliográficos de los científicos sociales (Bibliographic needs and usage of social scientists), cita investigaciones previas y afirma que los científicos sociales usan pocos materiales en lenguas extranjeras. Los rangos de variación en el uso de materiales en lengua inglesa (lengua materna de los sujetos estudiados) van del 87 al $98 \%$.

\section{Metodología}

Juan Samaja en su libro Epistemología y metodología (14) identifica tres componentes del proceso de investigación científica: el objeto o producto del proceso, las acciones investigativas o métodos y los medios o condiciones de realización. El primero es el conocimiento científico, resultante de la combinación de componentes teóricos y empíricos. El segundo comprende las acciones dirigidas al descubrimiento y a la validación del conocimiento. Y el último está constituido por los medios técnicos e institucionales en los que se desarrolla la investigación.

La combinación entre los componentes teóricos y empíricos está dada por los cursos de acción metodológica seguidos. Su núcleo reside en el diseño del objeto de estudio de la investigación que consiste en la elección de un modo de delimitar el objeto y trabajar sobre el sistema de matrices de datos definido (15).

Samaja sostiene que

«todos los datos de todas las investigaciones científicas de todas las disciplinas poseen esta estructura invariante que se puede llamar matriz de datos (...) [y que] todas las investigaciones científicas contienen datos de distinto tipo y de diferentes niveles de integración; no definen una sola matriz de datos, sino un conjunto de matrices que guardan entre sí relaciones lógico-metodológicas determinadas...» (14), p. 164-165.

Existen por lo menos tres niveles jerárquicos: 1) la matriz central en el nivel de anclaje sobre el que se enfoca la investigación, 2) la matriz de nivel subunitario (componentes de la unidad de análisis del nivel anterior), y 3) la matriz de nivel supraunitario (contextos de las unidades de análisis del nivel de anclaje). En los diferentes niveles pueden existir matrices coordinadas de la misma jerarquía.

La propuesta metodológica de Samaja (14) sustenta dos elementos de originalidad: por un lado, la presentación de una estructura cuatripartita de la matriz de datos que agrega los indicadores o esquemas indicadores a los tres ya conocidos (unidad de aná- 
lisis, variables y valores) y por el otro, la relación dialéctica que se configura entre las matrices mencionadas.

En esta investigación se ha llamado nivel de anclaje al que tiene como unidad de análisis el Acceso a la información, con las siguientes variables: procesos de acceso, medios formales de acceso y medios informales de acceso. La unidad de análisis de la matriz de nivel supraunitario es el proyecto de investigación en Humanidades o en Ciencias Sociales que da contexto al acceso a la información. Se diseñó una matriz coordinada a la matriz central, cuya unidad de análisis es la información de la investigación. El nivel subunitario está constituido por las variables de la matriz central: formato, obsolescencia, dispersión temática y lengua.

Para la recolección y el análisis de los datos se utilizó una estrategia de triangulación metodológica combinando técnicas cuantitativas y cualitativas de recopilación de datos, para poder enriquecer y ampliar el análisis y la interpretación de los mismos.

Con los antecedentes reunidos y considerando los aspectos que la investigación se proponía conocer, se elaboró un primer cuestionario de prueba. Se discutió extensamente cuál sería la población a estudiar y se decidió circunscribir el estudio a las unidades de investigación de la Facultad de Filosofía y Letras (UBA). La puesta a prueba del cuestionario se realizó entrevistando a los investigadores. Con la información obtenida se redactó un cuestionario definitivo que se aplicó para estudiar las características de los investigadores de la Facultad de Filosofía y Letras: procedencia, edad, sexo, nivel académico, idiomas que manejan; los datos de la investigación, la forma de trabajo y la función que cumplen en el grupo; utilización de las bibliotecas y consulta a los bibliotecarios; acceso a la bibliografía necesaria para la investigación o para la actualización permanente; tiempo dedicado a distintos aspectos de la tarea de investigación; obstáculos a la búsqueda de información; nivel de satisfacción frente a las colecciones; tipo de formato preferido y edad de los documentos; utilización de formatos alternativos y uso de computadoras.

Se distribuyeron los cuestionarios en las secretarías de 18 Institutos, Secciones y Centros de Investigación de la Facultad de Filosofía y Letras; a través de los secretarios tanto se repartieron como se reunieron los formularios completados por los investigadores. Sobre una población total estimada de 180 sujetos, se contestaron y procesaron 124 encuestas, cubriendo con éstas para Humanidades: Artes, Filosofía y Literatura; y para Ciencias Sociales: Ciencias Antropológicas, Ciencias de la Educación, Geografía, Historia y Bibliotecología. En Humanidades se encuestaron 53 investigadores en total: 13 de Filosofía, 30 de Literatura y 5 de Artes. En Ciencias Sociales se encuestaron 61 investigadores: 24 antropólogos, 12 bibliotecólogos, 8 especialistas en educación, 8 geógrafos y 9 historiadores.

Se realizaron entrevistas estructuradas a los bibliotecarios encargados de las colecciones y servicios de los diferentes centros de investigación, con el propósito de agregar otro punto de vista que enriqueciera la interpretación de los datos recolectados mediante las encuestas a los investigadores y el análisis de las citas de sus artículos. Para tal fin, fue diseñada una guía de entrevista y se volcaron los datos en tablas que facilitaron su análisis y comparación.

Se realizó un estudio de las referencias citadas en los trabajos publicados por los investigadores estudiados. Se recopilaron las publicaciones periódicas editadas por la Facultad de 1990 a 1996, lo que resultó en un número total de 499 artículos y 11.379 
referencias. Se diseñó una muestra estratificada tomando como criterio el área temática (Lengua y Literatura, Filosofía, Artes, Historia, Ciencias Antropológicas y Ciencias de la Educación). Se seleccionó al azar una cantidad proporcional al tamaño de los estratos resultando un total de 128 artículos, de los cuales se analizaron 3.449 referencias bibliográficas citadas. Se consideraron las siguientes variables: materia, formato, edad y lenguas. En los artículos redactados por los investigadores se analizó una sola variable, la materia, a fin de establecer vínculos con la materia de las referencias citadas y detectar relaciones de interdisciplinariedad entre artículos y referencias citadas. Se diseñó una base de datos relacional con el programa para procesamiento estadístico EPIINFO. Se realizaron diversas operaciones de análisis y síntesis de los datos.

En primer lugar, se discriminó entre fuentes y literatura secundaria, entendiendo por fuentes aquellas que constituyen el objeto de estudio o unidad de análisis de la investigación y cuyos resultados presenta el artículo analizado. Se consideró literatura secundaria al resto de la literatura citada, o sea, otras investigaciones relacionadas y el aparato crítico/teórico. Se buscó establecer qué porcentaje de fuentes se citan en Humanidades y en Ciencias Sociales esperando que éste fuera mayor en el primer caso porque, como sostiene Atkinson (16), cita y referencia coinciden.

Se realizó un análisis temático de la literatura secundaria citada a fin de compararla con los artículos analizados, con el objeto de lograr una medida de su interrelación temática. Esta fue expresada en porcentajes complementarios: autocita disciplinar cuando se cita literatura secundaria perteneciente a la misma disciplina y alocita disciplinar cuando la cita corresponde a otras disciplinas.

Las conclusiones obtenidas con las diversas técnicas implementadas fueron confrontadas y comparadas usando la estrategia de triangulación antes mencionada, lo que posibilitó su verificación y validación posterior.

\section{Resultados}

\subsection{Acceso a la información}

Los datos respecto de este punto provienen de las encuestas que se distribuyeron entre los investigadores de la Facultad de Filosofía y Letras (UBA). En los cuestionarios se diferenció entre las búsquedas de información necesaria para la investigación y de información para la actualización permanente en la especialidad.

Es posible tipificar la muestra de investigadores estudiada tanto en Humanidades como en Ciencias Sociales como universitarios dedicados a desarrollar una carrera académica, con una tendencia creciente a la obtención de postgrados. Un $29 \%$ de los encuestados en Humanidades ha obtenido el doctorado, mientras que en Ciencias Sociales, si bien sólo lo había hecho el $14 \%$, muchos de los investigadores estaban en proceso de obtenerlo. En ambas áreas disciplinares muy altos porcentajes manejan al menos dos idiomas extranjeros (inglés y francés). Dentro de las Humanidades, se destacan los investigadores de Literatura con un $45 \%$ que domina cuatro lenguas (inglés, francés, italiano y portugués).

En cuanto a la modalidad de trabajo, si bien hay ciertas disimilitudes entre las disciplinas de ambas áreas, se manifiesta una tendencia clara hacia la constitución de equipos de investigación. Por otra parte, la muestra presentó una interesante diversi- 
dad de funciones: directores de proyectos, auxiliares de investigación, graduados con becas para investigar y docentes/investigadores.

Todos los encuestados, salvo dos en el área de Humanidades e igual número en la de Ciencias Sociales, afirman frecuentar la biblioteca para buscar información. Si bien en ambos grupos predomina la frecuencia de asistencia de 1 a 2 veces por semana, ese porcentaje es algo mayor en Humanidades (66\%) que en Ciencias Sociales $(50 \%)$, donde la frecuencia de menos de una vez por semana alcanza el $33 \%$ a diferencia del $12 \%$ para las Humanidades. Mientras que en Ciencias Sociales un 5\% no sabe o no contesta las veces que concurre a la biblioteca, en Humanidades hay un $19 \%$ que lo hace de 3 a 4 veces por semana. Estos datos nos llevan a fijar una tendencia de uso más intensivo de la biblioteca por parte de los humanistas, lo cual es comprensible si consideramos las diferentes técnicas de recopilación de datos de las que disponen los científicos sociales fuera de la biblioteca, como, por ejemplo, el trabajo de campo. De todos modos, más del $70 \%$ de ambos grupos considera que las contribuciones de las bibliotecas a su investigación son importantes.

¿Cómo acceden los encuestados a la información para sus proyectos de investigación? En ambos casos, el recurso predominante es la consulta a los colegas y la lectura de revistas de la especialidad.

Tampoco se encuentran diferencias notables en el uso que ambos grupos hacen de las herramientas bibliograficas formales para sus investigaciones. Se trata, específicamente, de búsquedas retrospectivas para iniciar y mantener al día un estado de la cuestión sobre el tema que se indaga. En ambas áreas son más los que no usan bibliografías (73\% para Humanidades y $77 \%$ para Ciencias Sociales) que los que lo hacen $(17,18,19)$.

Para la actualización permanente, los especialistas en Humanidades prefieren examinar revistas y, en segundo término, consultar a los colegas; a la inversa, los científicos sociales indican recurrir primero a sus pares $y$, en segundo lugar, revisar las publicaciones periódicas.

Como herramientas de actualización permanente, las bibliografías ocupan un lugar intermedio en la preferencia de ambas áreas, pero este dato debe ser tomado con mucha precaución, dado que la gran mayoría de los investigadores cuando se les pregunta si consultan bibliografías, se refieren a las citas que aparecen en libros y artículos de revistas, y no a herramientas formales de acceso a la literatura especializada; esto se pudo comprobar con la pregunta siguiente del cuestionario donde se les solicitaba la mención de las herramientas bibliográficas utilizadas y donde, la mayoría de los investigadores no indicaba ningún título. En ambos campos y en menor medida, las preferencias subsiguientes se reparten en listas y catálogos editoriales, cursos y talleres y un modesto porcentaje $(10 \%)$, consulta las bibliotecas $(17,18,19)$.

De acuerdo con los datos recopilados, se puede deducir que las obras de referencia bibliográficas son, en general, poco usadas en ambos campos, menos en Ciencias Sociales que en Humanidades y que, en ambas áreas disciplinarias, son prácticamente desestimadas como herramientas de actualización permanente.

Estas respuestas fueron confirmadas por las entrevistas a los bibliotecarios, quienes manifestaron que los investigadores recurren a los fondos de la biblioteca para obtener el material recomendado por los colegas o citado en las revistas pero que, en general, no utilizan los catálogos de la biblioteca ni las herramientas bibliográficas disponibles para hacer búsquedas temáticas.

En ambos campos, los obstáculos más importantes que enfrentan los investigadores 
en su búsqueda de información, y que reconocen como tales, son la carencia de un número suficiente de libros y revistas y la pérdida de materiales. En Humanidades, además, se indica en tercer lugar el tiempo que transcurre entre el pedido y la recepción del material y, en cuarto término, localizar y obtener libros y revistas. En Ciencias Sociales el tercer obstáculo son los libros y revistas mal ubicados, seguido por la ausencia prolongada de los materiales en préstamo. Como se ve, los inconvenientes más importantes se derivan de carencias presupuestarias, pero el resto de los problemas se origina en ciertos defectos organizativos de las bibliotecas que serían fácilmente subsanables.

Como puede apreciarse en el caso estudiado, las diferencias en las modalidades de acceso en ambas áreas no son sustanciales ni para la investigación ni para la actualización permanente y las formas coinciden con las descritas en otros estudios (3, 4, 10, 11): existe una marcada preferencia por los canales informales y la biblioteca se utiliza como recurso para obtener materiales que no se pueden conseguir por otros medios (véase tabla I)

\subsection{Formato de los materiales}

En este punto contamos con tres fuentes de datos: los investigadores (según las encuestas), los bibliotecarios (de acuerdo con las entrevistas) y las citas (datos derivados de su análisis).

Tabla I

Modos de acceso a la información

\begin{tabular}{|c|c|c|}
\hline & Humanidades & Ciencias Sociales \\
\hline Investigación & $\begin{array}{l}\text { 1) Consulta a los colegas. } \\
\text { 2) Lectura y examen de revistas. }\end{array}$ & $\begin{array}{l}\text { 1) Consulta a los colegas. } \\
\text { 2) Lectura y examen de revistas. }\end{array}$ \\
\hline $\begin{array}{l}\text { Actualización } \\
\text { permanente }\end{array}$ & $\begin{array}{l}\text { 1) Lectura y examen de revistas. } \\
\text { 2) Consulta a los colegas. }\end{array}$ & $\begin{array}{l}\text { 1) Consulta a los colegas. } \\
\text { 2) Lectura y examen de revistas. }\end{array}$ \\
\hline $\begin{array}{l}\text { Utilización de la } \\
\text { biblioteca }\end{array}$ & $\begin{array}{l}\text { Propósito predominante: Obtención de } \\
\text { materiales previamente identificados. } \\
\text { Frecuencia: } \\
\text { 3-4 veces por semana, } 19 \% \\
\text { 1-2 veces por semana, } 66 \% \\
\text { Menos de } 1 \text { vez por semana, } 12 \% \\
\text { Sin respuesta, } 3 \%\end{array}$ & $\begin{array}{l}\text { Propósito predominante: Obtención de } \\
\text { materiales previamente identificados. } \\
\text { Frecuencia: } \\
3-4 \text { veces por semana, } 8 \% \\
1-2 \text { veces por semana, } 52 \% \\
\text { Menos de } 1 \text { vez por semana, } 35 \% \\
\text { Sin respuesta, } 5 \%\end{array}$ \\
\hline $\begin{array}{l}\text { Utilización de } \\
\text { bibliografías y } \\
\text { servicios secundarios }\end{array}$ & $27 \%$ & $23 \%$ \\
\hline Obstáculos al acceso & $\begin{array}{l}\text { 1. Carencia de suficiente cantidad de } \\
\text { libros y revistas. } \\
\text { 2. Pérdida del material. } \\
\text { 3. Largo tiempo de demora entre pe- } \\
\text { dido y recepción de los materiales. } \\
\text { 4. Serias dificultades para localizar y } \\
\text { obtener el material. }\end{array}$ & $\begin{array}{l}\text { 1. Carencia de suficiente cantidad de } \\
\text { libros y revistas. } \\
\text { 2. Pérdida del material. } \\
\text { 3. Libros y revistas mal ubicados. } \\
\text { 4. Períodos de préstamo a domicilio } \\
\text { demasiado prolongados. }\end{array}$ \\
\hline
\end{tabular}


La preferencia manifiesta de los investigadores con respecto a libros o revistas fue indagada en forma indirecta, dado que en la encuesta se les preguntó cómo distribuirían el presupuesto para adquisiciones de la biblioteca. De esos porcentajes asignados se puede deducir la mayor o menor importancia que se le da a ambos formatos. Así, en Humanidades un $43 \%$ prefiere los libros sobre las revistas, a la inversa un $23 \%$, y le asigna la misma importancia un $34 \%$. Mientras que en Ciencias Sociales los porcentajes son respectivamente del $31 \%$ para más libros, el $41 \%$ para más publicaciones periódicas, y el $28 \%$ para ambos por igual. Esto muestra una marcada preferencia de los humanistas por el formato libro sobre el de la publicación periódica y una tendencia inversa en las Ciencias Sociales, aunque son más en el área de Humanidades los que le asignan igual importancia a ambos formatos.

Según la opinión mayoritaria de los bibliotecarios de los centros de investigación de la Facultad, en las bibliotecas se consultan más las revistas que los libros.

En el análisis de citas se ve que en ambas áreas disciplinarias se prefiere ampliamente el formato libro seguido por el artículo de publicación periódica. En Humanidades, $62.05 \%$ vs. $22.22 \%$ y en Ciencias Sociales, $51.16 \%$ vs. $28.11 \%$ (véase tabla II).

Tabla II

Formato del material utilizado

\begin{tabular}{|l|c|r|r|r|r|r|}
\hline \multicolumn{1}{|c|}{ Formato } & Humanidades & $\%$ & $\begin{array}{c}\text { Ciencias } \\
\text { Sociales }\end{array}$ & $\%$ & Total & $\%$ \\
\hline Libros & 551 & 62,0 & 1.206 & 47,1 & 1.757 & 50,9 \\
Articulos de revistas & 197 & 22,2 & 778 & 30,4 & 975 & 28,3 \\
Diarios & 49 & 5,5 & 146 & 5,7 & 195 & 5,7 \\
Ponencias a congresos & 17 & 1,9 & 120 & 4,7 & 137 & 4,0 \\
Informes & 27 & 3,0 & 49 & 1,9 & 76 & 2,2 \\
Otros & 48 & 5,4 & 261 & 10,2 & 309 & 9,0 \\
\hline Total & 889 & 100,0 & 2.560 & 100,0 & 3.449 & 100,0 \\
\hline
\end{tabular}

Se ha notado que la cita a fuentes es significativa, independientemente del formato en el que se registren. En esta investigación se consideran fuentes a los textos o documentos originales, o a los datos primarios publicados (20). En Humanidades la cita a fuentes es del $35.66 \%$ del total de las referencias citadas y en Ciencias Sociales es del $21.33 \%$ (véase tabla III)

Tabla III

Relación fuentes-bibliografía en el material citado

\begin{tabular}{|l|c|c|c|c|}
\hline & Fuentes & Bibliografia & Total & $\%$ Fuentes \\
\hline Humanidades & 317 & 572 & 889 & 35,66 \\
Ciencias Sociales & 546 & 2.014 & 2.560 & 21,33 \\
\hline Total & 863 & 2.586 & 3.449 & 25,02 \\
\hline
\end{tabular}


De las diferentes fuentes de datos se desprende que habría una oposición entre las percepciones que tienen los investigadores y los bibliotecarios sobre la preferencia de uso de formatos y lo demostrado por el análisis de citas. Se presupone que dicha oposición no existe; en primer término, porque las bibliotecas han priorizado la adquisición de las revistas sobre los libros. Esto ha producido un núcleo de colecciones actualizadas de publicaciones periódicas pero no así de monografías. Por lo tanto, los investigadores, en forma particular o a través de los subsidios de investigación, adquieren los libros más actuales y no se suscriben a revistas. La consulta de éstas se hace en la biblioteca de investigación, de ahí la percepción de los bibliotecarios y la preferencia manifestada por los investigadores. Sin embargo, el análisis de citas comprueba una preferencia marcada por el formato libro en detrimento del artículo a la hora de escribir una contribución a la investigación.

De estos datos puede deducirse que los papeles principales que representa la revista en Ciencias Humanas son el de actualización permanente y el de herramienta bibliográfica porque permite rastrear citas relevantes en sus artículos y en su sección de reseñas de libros. Sin embargo, este uso tan importante de la publicación periódica no tiene por qué verse necesariamente reflejado en las citas, donde queda demostrado que el libro ocupa un lugar preeminente. Una vez más, se confirma que los datos derivados del análisis de citas deben tomarse con mucha precaución para decidir las adquisiciones en las bibliotecas (21), dado que dichos estudios no consideran los diferentes propósitos con los que se utilizan los materiales de estudio e investigación.

\subsection{Edad de los materiales utilizados}

Tal como se ha mostrado en las revisiones de la literatura sobre el tema $(3,4,12)$, los estudios de usuarios destacan que hay menor obsolescencia del material utilizado por los especialistas en Humanidades comparándola con la que se da en Ciencias Sociales.

En esta investigación se ha podido comprobar una tendencia similar. Es importante aclarar que, dentro de las citas para estudiar la edad de los materiales, se han excluido las fuentes, o sea, los documentos primarios objeto del análisis y el estudio de los investigadores.

Las citas a documentos secundarios (otras investigaciones y aportes teóricos y críticos) se concentran en gran medida en el siglo $\mathrm{xx}$ y el resto en el XIX. Esto se verifica en dos períodos: 1900-1949 y 1980-1995, dado que, comparativamente dentro de cada intervalo, hay un mayor porcentaje de citas de Humanidades en el primer período, mientras que en el segundo el porcentaje mayor es el de Ciencias Sociales. En el período intermedio, 1950-1979, los porcentajes son relativamente parejos en ambas áreas (véase tabla IV)

Estos datos son importantes para que las bibliotecas puedan definir con un mayor grado de certeza sus políticas de descarte, de preservación y de conservación de los materiales; ambos temas son muy problemáticos y sensibles y no siempre han merecido la debida atención de parte de las autoridades de las instituciones universitarias. 
Tabla IV

Referencias citadas según período temporal

\begin{tabular}{|c|c|c|c|c|c|c|c|c|}
\hline & \multicolumn{4}{|c|}{ Total de referencias citadas } & \multicolumn{4}{|c|}{ Referencias citadas excl. fuentes } \\
\hline & \multicolumn{2}{|c|}{ Humanidades } & \multicolumn{2}{|c|}{ Ciencias Sociales } & \multicolumn{2}{|c|}{ Humanidades } & \multicolumn{2}{|c|}{ Ciencias Sociales } \\
\hline & $\begin{array}{l}N .^{\circ} \text { de } \\
\text { citas }\end{array}$ & $\%$ & $\begin{array}{l}N .^{\circ} \text { de } \\
\text { citas }\end{array}$ & $\%$ & $\begin{array}{l}N .^{\circ} \text { de } \\
\text { citas }\end{array}$ & $\%$ & $\begin{array}{l}N .^{\circ} \text { de } \\
\text { citas }\end{array}$ & $\%$ \\
\hline Antes de 1900 & 54 & 6,1 & 371 & 14,5 & 1 & 0,7 & 7 & 2,0 \\
\hline $1900-1949$ & 260 & 29,2 & 261 & 10,2 & 33 & 12,5 & 18 & 7,0 \\
\hline $1950-1979$ & 251 & 28,2 & 640 & 25,0 & 91 & 36,3 & 189 & 29,5 \\
\hline $1980-1995$ & 324 & 36,5 & 1.288 & 50,3 & 163 & 50,4 & 792 & 61,5 \\
\hline Total & 889 & 100,0 & 2.560 & 100,0 & 288 & 100,0 & 1.006 & 100,0 \\
\hline
\end{tabular}

\subsection{Materias citadas}

El propósito de analizar temáticamente las referencias citadas ha sido estudiar el grado de cita a la propia disciplina (porcentaje de autocita disciplinar) y a otras diferentes (porcentaje de alocita disciplinar). Los resultados pueden guiar las políticas de centralización o descentralización de las colecciones de las bibliotecas de investigación en las áreas consideradas por esta investigación.

Para estudiar las materias de las referencias citadas se tomaron algunas decisiones metodológicas. En primer lugar, utilizar categorías temáticas generales y en segundo, las especialidades que se incluirían en cada área. En Humanidades se consideraron tres: Artes (Artes Plásticas, Artes del Espectáculo y Música), Filosofía y Lengua y Literatura. En este último caso se formó un solo bloque con Literatura y Lingüística, dado que ambas son especializaciones de una única carrera. En Ciencias Sociales se incluyeron cinco: Ciencias Antropológicas -que abarca Antropología Sociocultural, Arqueología, Antropología Biológica y Folklore-, Historia, Ciencias de la Educación, más dos campos híbridos (22): Etnohistoria y Etnolingüística. Por la naturaleza de estos dos últimos casos se consideró cita a la propia disciplina las citas de Etnohistoria a Ciencias Antropológicas e Historia, y de Etnolingüística a Ciencias Antropológicas y Lingüistica.

En general, la autocita disciplinar es algo mayor en Ciencias Sociales. En Humanidades. el promedio es de 38,7. Filosofía tiene el más alto porcentaje mientras que Artes el menor, en tanto que Literatura ocupa un lugar intermedio. Esto quizás pueda deberse a que la carrera de Artes, tal como se ha orientado su enseñanza en la Facultad, se nutre de marcos teóricos de otras disciplinas (véase tabla V). Para Humanidades no se han encontrado estudios similares en la literatura especializada que permitan establecer comparaciones.

En Ciencias Sociales el porcentaje de autocita disciplinar es 50,74. Porcentaje que se aproxima bastante al 58\% indicado por $\mathrm{Li} \mathrm{(15)} \mathrm{Todas} \mathrm{las} \mathrm{disciplinas} \mathrm{se} \mathrm{encuentran}$ cercanas a esta media, salvo Etnolinguiística que alcanza el $82 \%$. Porcentaje que debe tomarse con precaución, dado que podría atribuirse al azar, por el reducido tamaño de la muestra para esta especialidad (véase tabla VI). 
Tabla V

Autocita y alocita disciplinar en Humanidades

\begin{tabular}{|l|c|c|c|c|c|c|}
\hline \multicolumn{1}{|c|}{ Disciplina } & $\begin{array}{c}\text { Autocitar } \\
\text { disciplinar }\end{array}$ & $\begin{array}{c}\text { \% Autocita } \\
\text { disciplinar }\end{array}$ & $\begin{array}{c}\text { Alocita } \\
\text { disciplinar }\end{array}$ & $\begin{array}{c}\text { \% Alocita } \\
\text { disciplinar }\end{array}$ & Total & $\begin{array}{c}\text { \% } \\
\text { Total }\end{array}$ \\
\hline Arte & 58 & 21,1 & 223 & 79,0 & 281 & 100,0 \\
Filosofía & 90 & 58,6 & 66 & 41,5 & 156 & 100,0 \\
Lengua y Literatura & 196 & 43,3 & 256 & 56,7 & 452 & 100,0 \\
\hline Total & 344 & 38,7 & 545 & 61,3 & 889 & 100,0 \\
\hline
\end{tabular}

Tabla VI

Autocita y alocita disciplinar en Ciencias Sociales

\begin{tabular}{|l|c|c|c|c|c|c|}
\hline \multicolumn{1}{|c|}{ Disciplina } & $\begin{array}{c}\text { Autocitar } \\
\text { disciplinar }\end{array}$ & $\begin{array}{c}\text { \% Autocita } \\
\text { disciplinar }\end{array}$ & $\begin{array}{c}\text { Alocita } \\
\text { disciplinar }\end{array}$ & $\begin{array}{c}\text { \% Alocita } \\
\text { disciplinar }\end{array}$ & Total & $\begin{array}{c}\% \\
\text { Total }\end{array}$ \\
\hline Ciencias Antropológicas & 721 & 52,2 & 660 & 47,8 & 1.381 & 100,0 \\
Etnohistoria & 69 & 52,9 & 63 & 47,1 & 132 & 100,0 \\
Historia & 231 & 46,2 & 268 & 53,8 & 499 & 100,0 \\
Etnolingüística & 63 & 82,3 & 13 & 17,7 & 76 & 100,0 \\
Ciencias de la Educación & 214 & 45,6 & 258 & 54,4 & 472 & 100,0 \\
\hline Total & 1.298 & 50,7 & 1.262 & 49,3 & 2.560 & 100,0 \\
\hline
\end{tabular}

Aun las citas efectuadas a otras disciplinas (alocita disciplinar) se mantienen dentro del campo de las Humanidades y las Ciencias Sociales indicando una fuerte interrelación entre sus especialidades. Ciencias Antropológicas constituye, en algún sentido, un caso especial porque dentro de éstas, Arqueología, Antropología Biológica, Antropología Médica, etc. citan materiales de otras ciencias fuera de los límites de esas áreas.

Estos resultados llevan a coincidir con la recomendación de Broadus (7) para $\mathrm{Hu}$ manidades, respecto de la centralización de las colecciones. En la misma dirección, dada la dispersión geográfica de las bibliotecas de investigación de la Facultad de Filosofía y Letras, sería más conveniente para los investigadores que se reunieran las colecciones en un solo lugar para facilitar la consulta interdisciplinaria y superar los inconvenientes logísticos que suelen plantear los préstamos entre institutos. Por otra parte, la implementación definitiva de una red que reúna los fondos dispersos en diferentes centros, no alcanzaría a resolver el problema de la dispersión geográfica de los mismos.

\subsection{Idioma de los materiales}

En las encuestas, los entrevistados manifiestan su capacidad para manejar idiomas extranjeros: de dos a cuatro en Humanidades y dos en Ciencias Sociales.

Sin dudar de la veracidad de estos datos, las citas analizadas demuestran una pre- 
ferencia por el idioma español: en Humanidades $76.5 \%$ y en Ciencias Sociales $73.5 \%$. El segundo idioma citado varía para cada área: italiano y francés en la primera e inglés en la segunda (véase tabla VII).

Tabla VII

Idioma del material utilizado

\begin{tabular}{|l|c|c|c|c|c|}
\hline Idioma & Humanidades & $\%$ & Ciencias Sociales & $\%$ & Total \\
\hline Español & 680 & 76,5 & 1.882 & 73,5 & 2.562 \\
Inglés & 51 & 5,7 & 450 & 17,6 & 501 \\
Francés & 64 & 7,2 & 109 & 4,3 & 173 \\
Italiano & 71 & 8,0 & 40 & 1,6 & 111 \\
Portugués & 1 & 0,1 & 27 & 1,1 & 28 \\
Alemán & 10 & 1,1 & 19 & 0,7 & 29 \\
Multilingiue & 5 & 0,6 & 5 & 0,2 & 10 \\
Latín & 4 & 0,5 & 4 & 0,2 & 8 \\
Griego & 2 & 0,2 & 0 & 0,0 & 2 \\
Otro & 1 & 0,1 & 24 & 0,9 & 25 \\
\hline Total & 889 & 100,0 & 2.560 & 100,0 & 3.449 \\
\hline
\end{tabular}

Estos porcentajes, que se aproximan bastante al $87 \%$ indicado por $\mathrm{Li}$ (12) para Ciencias Sociales, y al $78 \%$ registrado por Cullars $(8,9)$ para Humanidades, corroborarían la tendencia de los investigadores a manejarse preferentemente con materiales redactados en su propia lengua o traducidos a ésta. Una vez más, los datos permiten guiar las adquisiciones de las unidades de información: los documentos fuente en sus idiomas originales o en versiones bilingües (cuando las hay); los materiales críticos, teóricos o históricos preferirlos en español o en buenas traducciones cuando están en lenguas extranjeras.

\section{Conclusiones}

En primer lugar se puede afirmar que no existen diferencias sustantivas en las conductas de búsqueda de información entre los investigadores de Humanidades y los de Ciencias Sociales. En ambas áreas se hace un uso limitado de las herramientas bibliográficas y de los servicios de información secundarios. Se recurre a la biblioteca porque esta tiene más material y lo organiza para facilitar su ubicación; sin embargo, se la utiliza principalmente para localizar la literatura previamente identificada por otros medios. Las revistas son instrumentos tanto de búsqueda retrospectiva de información, mediante el rastreo de citas, como de actualización permanente, a través de la lectura de sus artículos. La consulta a colegas y expertos es una estrategia de búsqueda muy apreciada y frecuentemente utilizada.

Respecto del formato, ambos campos disciplinares citan más libros que artículos de revistas en sus trabajos de investigación. Hay un pequeño matiz diferencial, dado que menos de la cuarta parte de las citas en Humanidades remiten a artículos de revistas, mientras que en Ciencias Sociales lo hace algo más del $25 \%$. Por su parte, las 
fuentes objeto de estudio son más utilizadas en Humanidades que en Ciencias Sociales. Tal como ya se advirtió antes, esto no indica en forma excluyente cuál es el formato preferido, dado que libros y revistas cumplen funciones diversas y satisfacen necesidades diferentes de los investigadores.

En cuanto a la edad de los materiales, cuando se excluyen las fuentes objeto de estudio, en ambas áreas la mitad o algo más (Ciencias Sociales, 61.5\%) se concentran en el período 1980-1995. La otra mitad (o algo menos en Ciencias Sociales) se distribuye prácticamente toda dentro del siglo $\mathrm{xX}$, concentrándose en la segunda mitad de la centuria y disminuyendo sustancialmente en la primera. Si bien los porcentajes diferenciales rondan el $10 \%$, podría afirmarse que la literatura crítica/teórica tiene algo más de vigencia temporal en Humanidades.

Cuando se observan los datos referidos a las materias citadas, haciendo la salvedad de que esto varía mucho entre disciplinas, en líneas generales se desprende que la alocita disciplinar es mayor en Humanidades que en Ciencias Sociales: en estas últimas se equilibra la autocita con la alocita disciplinar. De todos modos, si bien no es el objetivo de este artículo, este punto debería refinarse analizando las materias citadas dentro del conjunto de las Humanidades o de las Ciencias Sociales -citas a otras disciplinas humanísticas o sociales. Si bien la alocita es exógena respecto de la propia disciplina podría ser endógena dentro del campo disciplinar.

Por último, los datos reunidos y analizados evidencian la marcada preferencia por leer en la propia lengua, cuando existen materiales originales o traducidos que satisfacen ese requisito.

En este punto, si confrontamos los resultados obtenidos con esta investigación con los datos aportados en la sección 2. Estado de la cuestión, podemos contestar nuestra pregunta inicial diciendo que es correcto transferir los estudios de usuarios de países con sistemas y servicios de información avanzados a otros, como la Argentina, que tienen un nivel inferior de desarrollo de los mismos. Esto nos permite confirmar la segunda cuestión planteada inicialmente: a pesar de la calidad y cantidad de recursos, existe un substrato común que caracteriza al investigador en Humanidades y en Ciencias Sociales en sus actividades de acceso a la información, en sus preferencias y en sus necesidades informativas, independientemente del lugar donde desarrolla sus tareas y de las facilidades materiales de las que dispone. Ese substrato hay que relacionarlo con la conducta individual enfrentada a la situación cognoscitiva, lo que llevaría a preguntarse si, realmente, es adecuado buscar diferencias sustantivas profundas entre los individuos dada su pertenencia a una u otra disciplina. Una vez más, se demuestra que resolver un problema lleva a plantearse otros cada vez más sustantivos y difíciles. Sin embargo, tal como lo afirmaban Dervin y Nilan (23), en su ya clásica revisión, las respuestas no están en los sistemas sino en los usuarios.

\section{Nota}

(*) Modos de acceso y utilización de la bibliografía en el campo de las Humanidades y las Ciencias Sociales (FI 172). Programa de Investigación UBACYT 1994-97 (Universidad de Buenos Aires). Directora: Susana Romanos de Tiratel; integrantes: María Cristina Cajaraville, Virginia Garrote y Estela Tolosa. 


\section{Bibliografía}

1. CIRIGLIANO, G. F. J. La conducta informativa en universitarios argentinos: investigación sobre la habilidad y capacidad de los jovenes graduados universitarios para manejar y utilizar las fuentes de información bibliográficas. Buenos Aires: Centro de Investigaciones Bibliotecológicas, Facultad de Filosofía y Letras, Universidad de Buenos Aires, 1971. (Investigaciones no. 1). Como continuación de esta investigación se desarrolló otro proyecto, Investigación sobre la capacidad de los estudiantes avanzados de las universidades estatales argentinas para la investigación bibliográfica, cuyos datos y conclusiones permanecen inéditos y depositados en el Instituto de Investigaciones Bibliotecológicas (Facultad de Filosofía y Letras, UBA).

2. BABINI, D. Política nacional de información. 1982, Tesis de Doctorado otorgada por la Universidad de El Salvador. Buenos Aires.

3. WATSON-BOONE, R. The Information Needs and Habits of Humanities Scholars. $R Q$, 1994, vol. 34, pp. 203-216.

4. STONE, S. Humanities Scholars: Information Needs and Uses. Journal of Documentation, 1982, vol. 38, pp. 292-313.

5. BLAZEK, R. y AVERSA, E. The Humanities. (4." ed.). Englewood, Col.: Libraries Unlimited, 1994.

6. BROADUS, R. N. The Range of Subject Literatures Used by Humanities Scholars. Collection Management, 1990, vol. 12, pp. 61-68.

7. BROADUS, R. N. Information Needs of Humanities Scholars: a Study of Requests Made at the National Humanities Center. Library \& Information Science Research, 1987, vol. 9, pp. 113-129.

8. CULlARS, J. Citation Characteristics of French and German Literary Monographs. Library Quarterly, 1989, vol. 59, pp. 305-325.

9. CULLARS, J. Citation Characteristics of Monographs in the Fine Arts. Library Quarterly, 1992, vol. 62, pp. 325-342.

10. FOLSTER, M. B. Information Seeking Patterns: Social Sciences. The Reference Librarian, 1995, núms. 49/50, pp. 83-93.

11. BAXTER, P. M. A View of Academics' Literature Search Methods: The Case of the Social Sciences and its Implications for Students. The Reference Librarian, 1990, núms. 27/28, pp. 419-431.

12. LI, T. Social Science Reference Sources: A Practical Guide (2. ${ }^{a}$ ed.). New York: Greenwood Press, 1990.

13. WEBB, W. H. Sources of Information in the Social Sciences (3. ${ }^{a}$ ed.). Chicago: American Library Association, 1986.

14. SAMAJA, J. Epistemología y metodología (2ª ed.). Buenos Aires: Eudeba, 1994.

15. WHITE, H. D. Reference Books, Databases, and the Repertoire. En: WHITE, H. D.; BATES, M. J. y WILSON, P. For Information Specialists: Interpretations of Reference and Bibliographic Work. Norwood, NJ: Ablex, 1992, pp. 27-78. Este autor aplica el concepto de matriz de datos al análisis de la estructura de las obras de referencia.

16. ATKINSON, R. Humanities Scholarship and the Research Library. Library Resources and Technical Services, 1994, vol. 39, pp. 79-84.

17. ROMANOS DE TIRATEL, S. Modos de acceso y utilización de la bibliografía en los procesos de producción de información. Trabajos presentados a la $29^{a}$ Reunión Nacional de Bibliotecarios. La biblioteca y la información en la transformación social, económica y cultural, 1995, abril 3-7, Buenos Aires, pp 51-56.

18. GRUPO DE ESTUDIO DE USUARIOS. Modos de acceso a la información de los investigadores de la Universidad; directora: Susana Romanos de Tiratel. Trabajos presen- 
tados al $1^{\circ}$ Encuentro Nacional. La universidad como objeto de investigación, 1995, septiembre 28-29, Buenos Aires, 19 p.

19. HERNÁNDEZ SALAZAR, P., coord. Seminario latinoamericano sobre formación de usuarios de la información y los estudios de usuarios; participantes: Saray Córdoba González, Ma. Cristina Pérez Giffoni, Susana Romanos de Tiratel, Martha Sabelli de Louzao. México: Universidad Nacional Autónoma de México, 1997.

20. ROMANOS DE TIRATEL, S. Guía de fuentes de información especializadas: Humanidades y Ciencias Sociales. Buenos Aires: EB publicaciones, 1996.

21. FALCATO, P. Evaluación administrativa de publicaciones periódicas en una biblioteca argentina especializada multidisciplinaria de investigación. Buenos Aires: CID-INTI, 1993.

22. DOGAN, M. y PAHRE, R. Las nuevas ciencias sociales. México: Grijalbo, 1993.

23. DERVIN, B. y NILAN, M. Information Needs and Uses. En: Annual Review of Information Science and Technology (ARIST), 1986, vol. 21, pp. 3-33. 